\title{
Affective Engineering in Application to Bi-Level Human Migration Models
}

\author{
Vyacheslav V. Kalashnikov, Nataliya I. Kalashnykova, \\ Yazmín G. Acosta Sánchez and Vitaliy V. Kalashnikov Jr
}

\begin{abstract}
In this paper, we develop a bi-level human migration model using the concepts of affective engineering (Kansei Engineering) and conjectural variations equilibrium (CVE). In contrast to previous existing works, we develop a bi-level programming model in a natural form. The upper level agents are municipalities of competing locations, whose strategies are investments into the infrastructures of the locations (cities, towns, etc.). These investments aim at making the locations more attractive for both residents and potential migrants from other locations, which clearly demands affective engineering tools. At the lower level of the model, the present residents (grouped into professional communities) are also potential migrants to other locations. They make their decision where to migrate (if at all) by comparing the expected values of the utility functions of the outbound
\end{abstract}

\author{
V. V. Kalashnikov $(\bowtie)$ \\ Tecnológico de Monterrey (ITESM, Campus Monterrey), \\ Ave. Eugenio Garza Sada 2501 Sur, 64849 Monterrey, NL, Mexico \\ e-mail: kalash@itesm.mx; slavkamx@mail.ru \\ V. V. Kalashnikov \\ Central Economics and Mathematics Institute (CEMI), \\ Russian iAcademy of Sciences (RAS), Nakhimovsky pr. 47, Moscow, Russian Federation \\ V. V. Kalashnikov \\ Sumy State University, Rimsky-Korsakov st. 2, Sumy 40007, Ukraine \\ N. I. Kalashnykova · Y. G. Acosta Sánchez \\ Universidad Autónoma de Nuevo León (UANL), \\ Ave. Universidad S/N, 66450 San Nicolás de los Garza, NL, Mexico \\ e-mail: nkalash2009@gmail.com \\ Y. G. Acosta Sánchez \\ e-mail: lic_acosta9@hotmail.com \\ V. V. Kalashnikov Jr \\ Graduate School of Economics, Universidad Autónoma de Nuevo León (UANL), \\ Campus Mederos, Ave. Lázaro Cárdenas 3600, 64890 Monterrey, NL, Mexico \\ e-mail: kalashnikov_de@yahoo.de
}


and inbound locations, estimated by taking into account their group's conjectures concerning equilibrium migration flows between the involved locations. The utility functions reflect the affective engineering technique because their values are based on the potential migrants' affection to the target locations. Applying a special technique to verify the consistency of the conjectures (influence coefficients), the existence and uniqueness results for the consistent conjectural variations equilibrium (CCVE) are established.

Keywords Bi-level human migration model - Kansei (Affection) utility functions • Variational inequality formulation - Consistency criterion - Consistent conjectural variations equilibrium

\section{Introduction}

Migration problems have been actively studied in many countries throughout the world, as the information/migration prediction data are extremely useful on a large economic scale. Migration prediction data can stipulate development of facilities necessary to advance employment, education, and ecology. Reciprocally, locations with more advanced infrastructure, higher employment capacities, ecologically friendly environments, etc., can generate the affection of the inhabitants and thus attract more potential migrants. However, overloaded housing/infrastructure facilities may reduce the comfort in everyday life, thus contradicting the Kansei engineering principles. Therefore, one can expect a trade-off in the investments into a locations infrastructure, balanced at a conjectural variations equilibrium state.

Various migration theories have been developed over time. In a shorter historical aspect, however, one may rely on the excellent fundamental survey Akkoyunlu and Vickerman [1].

In the works by Bulavsky and Kalashnikov [2,3], a new array of conjectural variations equilibria (CVE) was introduced and investigated in which the influence coefficients of each agent affected the structure of the Nash equilibrium. In particular, constant conjectural influence factors were used in the human migration model examined in [4]. More precisely, the potential migration groups were taking into account not only the current difference between the utility function values at the destination and original locations but also the possible variations in the utility values implied by the change of population volume due to the migration flows. These conjectured variations could be described with an aid of the so-called influence coefficients. In other words, we did not consider a perfect competition but rather a generalized Cournot-type model (in contrast to the classic Cournot model).

In their previous papers $[5,6]$, the authors extended the latter model to the case where the conjectural variation coefficients may not only be constants but also functions of the total population at the destination and of the group's fraction in it. Moreover, we allow these functions to take distinct values at the abandoned location and at the destination, which should elevate the models flexibility. As an experimental 
verification of the proposed model, we developed a specific form of the model based upon relevant population data of a three-city agglomeration at the boundary of two Mexican states: Durango (Dgo.) and Coahuila (Coah.). Specifically, we considered the 1980-2005 dynamics of population growth in three cities-Torreón (Coah.), Gómez Palacio (Dgo.), and Lerdo (Dgo.) — and proposed utility functions of three various types for each of the three cities. To our knowledge, these types of utility functions were not used in previous literature dealing with the human migration model. After collecting the necessary information about the average movement and transportation (i.e., migration) costs for each pair of cities, we applied the above-mentioned human migration model to this example. Numerical experiments were conducted, with interesting results concerning the probable equilibrium states.

The novel approach of the recent paper by Kalashnikov et al. [7] lies in the proposed definitions of consistent conjectures and in the outskirt of possible ways to calculate the consistent conjectures and the related consistent conjectural variation equilibrium state (CCVES).

Motivated by the ideas of bi-level structures of migration processes (the upper level competition among municipalities and the lower level equilibrium among the potential migrants), we proposed a new (bi-level) formulation of the human migration model. Under general enough assumptions, we also proved the existence of solutions to the bi-level program. The results of the numerical experiments (which are still underway) will only be outlined.

Sections 2 and 3, in primarily following the previous papers by Kalashnikov et al. [5, 6] and Kalashnikov et al. [7], describe the proposed bi-level human migration model, define the conjectural variation equilibrium at the lower level, and cite Theorems 3.1 and 3.2 from [5, 6], which establish the existence and uniqueness of the lower level equilibrium as a solution of an appropriate variational inequality problem. The consistency of the conjectures and the existence of the corresponding bi-level equilibrium are discussed in Sect. 4. In contrast to the previous paper [8], here we extend the lower lever (and thus, also the upper level) utility functions from linear to quadratic ones. The conclusions (Sect. 5), acknowledgments, and the list of references complete the paper.

\section{Problem Statement and Preliminaries}

Similar to [4-6], consider a closed economy with:

- $n$ locations, denoted by $i$;

- $K$ classes of population, denoted by $k$;

- $\bar{Q}_{i}^{k}$ initial fixed population of class $k$ in location $i$;

- $Q_{i}^{k}$ final population of class $k$ in location $i$;

- $s_{i j}^{k}$ migration flow of class $k$ from origin $i$ to destination $j$;

- $c_{i j}^{k}\left(s_{i j}^{k}\right)=b_{i j}^{k} s_{i j}^{k}+\frac{1}{2} a_{i j}^{k}\left(s_{i j}^{k}\right)^{2}$ migration cost for residents from group $k$ moving from location $i$ to location $j$. 
Assume that the migration cost reflects not only the cost of physical movement but also the personal and psychological (affection) cost as perceived by a class when moving between locations. The utility $u_{i}^{k}$ (attractiveness of location $i$ as perceived by class $k$ ) depends on the population at destination, that is, $u_{i}^{k}=u_{i}^{k}\left(Q_{i}^{k}\right)$. This assumption is quite natural: indeed, in many cases, the cities with higher population provide much more possibilities to find a job, better medical service and household facilities, a developed infrastructure, etc., which is readily described by the principles of Kansei Engineering. On the other hand, when the infrastructure development lags behind the modern city demands, the higher population may lead to certain decrease in the living standards, in the inhabitants' affection to their place, and hence, of the affection utility values.

These affection utility functions also incorporate parameters reflecting the scale of investments made by the location's authorities in order to improve the infrastructure, employment capacities, household construction, power supply, and so on, based upon the principles of Kansei Engineering. Exactly these amounts of investment play the role of the municipality strategies in the game at the upper level.

First, we describe the lower level problem. The conservation of flow equations, given for each class $k$ and each location $i$, and the inequalities forbidding repeated or chain migration are listed below:

$$
Q_{i}^{k}=\bar{Q}_{i}^{k}+\sum_{j \neq i} s_{j i}^{k}-\sum_{j \neq i} s_{i j}^{k}, \quad i=1, \ldots, n,
$$

and

$$
\sum_{j \neq i} s_{i j}^{k} \leq \bar{Q}_{i}^{k}, \quad i=1, \ldots, n,
$$

with $s_{i j}^{k} \geq 0, \forall k=1, \ldots, K ; j \neq i$. Denote the problem's feasible set by

$$
M=\{(Q, s) \mid s \geq 0,(Q, s) \text { satisfies (1) - (2) }\} .
$$

Equation (1) states that the population of class $k$ at location $i$ is determined by the initial population of class $k$ at location $i$ plus the migration flow into $i$ of that class minus the migration flow out of $i$ for the same class. Equation (2) postulates that the flow out of $i$ by migrants of group $k$ cannot exceed the initial population of group $k$ at $i$, because no chain migration is allowed in this model.

Assume that the migrants are rational and affection-motivated and that migration continues until no individual has any affection to the target location and thus any incentive to move, since a unilateral decision will no longer yield a positive net gain (the gain in the expected affection utility value minus the migration cost).

In order to extend the human migration model from [4], here we introduce the following concepts.

Definition 1 Let $w_{i j}^{k+} \geq 0$ be an influence coefficient taken in account by an individual of class $k$ considering a possibility of moving from $i$ to $j$. This coefficient is defined by his/her assumption that after the movement of $s_{i j}^{k}$ individuals of class $k$ from $i$ to $j$ the total population of class $k$ at $j$ equals: 


$$
\bar{Q}_{j}^{k}+w_{i j}^{k+} s_{i j}^{k}
$$

At the same time, let $w_{i j}^{k-} \geq 0$ be an influence coefficient conjectured by an individual of group $k$ planning to move from $i$ to $j$, determined by the assumption that after the movement of $s_{i j}^{k}$ individuals, the total population of class $k$ in $i$ will remain

$$
\bar{Q}_{i}^{k}-w_{i j}^{k-} s_{i j}^{k}
$$

We accept the following assumptions concerning the affection utility functions and expected variations in the utility values:

A1. The affection utility $u_{i}^{k}=u_{i}^{k}\left(Q_{i}^{k}\right)$ is a monotone decreasing and continuously differentiable function.

A2. Each person of group $k$, when considering his/her possibility of moving from location $i$ to location $j$, takes into account not only the difference in the affection utility values at the initial location and the destination, but also both the expected (negative) increment of the affection value at the destination $j$ :

$$
s_{i j}^{k} w_{i j}^{k+} \frac{\partial u_{j}^{k}}{\partial Q_{j}^{k}},
$$

and the expected (positive) affection utility value increment in the abandoned location $i$ :

$$
-s_{i j}^{k} w_{i j}^{k-} \frac{\partial u_{i}^{k}}{\partial Q_{i}^{k}} .
$$

\section{Definition of Equilibrium}

In this section, we will define what we understand as the conjectural variations equilibrium (CVE) both at the lower and the upper level of the new migration model.

\subsection{Definition of Equilibrium at the Lower Level}

At the lower level, we use the same concept of CVE as defined in our previous works [5-7].

Definition 2 A multiclass population and flow pattern $\left(Q^{*}, s^{*}\right) \in M$ are the equilibrium at the lower level, if for each class $k=1, \ldots, K$, and for every pair of locations $(i, j), i, j=1, \ldots, n ; i \neq j$, the following relationships hold: 


$$
u_{i}^{k}-s_{i j}^{k *} w_{i j}^{k-} \frac{\partial u_{i}^{k}}{\partial Q_{i}^{k}}\left(Q^{*}\right)+b_{i j}^{k}+a_{i j}^{k} s_{i j}^{k *}\left\{\begin{array}{l}
=u_{j}^{k}+s_{i j}^{k *} w_{i j}^{k+} \frac{\partial u_{j}^{k}}{\partial Q_{j}^{k}}\left(Q^{*}\right)-\lambda_{i}^{k}, \text { if } s_{i j}^{k *}>0 \\
\geq u_{j}^{k}+s_{i j}^{k *} w_{i j}^{k+} \frac{\partial u_{j}^{k}}{\partial Q_{j}^{k}}\left(Q^{*}\right)-\lambda_{i}^{k}, \text { if } s_{i j}^{k *}=0
\end{array}\right.
$$

and

$$
\lambda_{i}^{k}\left\{\begin{array}{l}
\geq 0, \text { if } \sum_{\ell \neq i} s_{i j}^{k *}=\bar{Q}_{i}^{k} \\
=0, \text { if } \sum_{\ell \neq i} s_{i j}^{k *}<\bar{Q}_{i}^{k}
\end{array}\right.
$$

In order to proceed with the equilibrium existence and uniqueness results, we need an extra assumption to hold.

A3. We assume that the influence coefficients are functions depending upon the current population at the location in question and the migration flow from location $i$ to location $j$, satisfying the following conditions:

$$
s_{i j}^{k} w_{i j}^{k+}(Q, s)=v_{i j}^{k+} s_{i j}^{k}+\sigma_{i j}^{k+} Q_{j}^{k},
$$

and

$$
s_{i j}^{k} w_{i j}^{k-}(Q, s)=v_{i j}^{k-} s_{i j}^{k}+\sigma_{i j}^{k-} Q_{i}^{k},
$$

where

$$
v_{i j}^{k \pm} \geq 0, \sigma_{i j}^{k \pm} \geq 0, k=1, \ldots, K ; i \neq j .
$$

Taking into account assumption $\mathrm{A} 3$ and omitting for shortness the argument $Q^{*}$ in the utility functions, we turn (8) into:

$$
\begin{aligned}
& u_{i}^{k}-s_{i j}^{k *} v_{i j}^{k-} \frac{\partial u_{i}^{k}}{\partial Q_{i}^{k}}+\sigma_{i j}^{k-} Q_{i}^{k *} \frac{\partial u_{i}^{k}}{\partial Q_{i}^{k}}+b_{i j}^{k}+a_{i j}^{k} j_{i j}^{k *}=u_{j}^{k}+s_{i j}^{k *} v_{i j}^{k+} \frac{\partial u_{j}^{k}}{\partial Q_{j}^{k}}+\sigma_{i j}^{k+} Q_{j}^{k *} \frac{\partial u_{j}^{k}}{\partial Q_{j}^{k}}-\lambda_{i}^{k}, \\
& \text { if } \quad s_{i j}^{k *}>0 ;
\end{aligned}
$$

and

$$
\begin{aligned}
& u_{i}^{k}-s_{i j}^{k *} v_{i j}^{k-} \frac{\partial u_{i}^{k}}{\partial Q_{i}^{k}}+\sigma_{i j}^{k-} Q_{i}^{k *} \frac{\partial u_{i}^{k}}{\partial Q_{i}^{k}}+b_{i j}^{k}+a_{i j}^{k} s_{i j}^{k *} \geq u_{j}^{k}+s_{i j}^{k *} v_{i j}^{k+} \frac{\partial u_{j}^{k}}{\partial Q_{j}^{k}}+\sigma_{i j}^{k+} Q_{j}^{k *} \frac{\partial u_{j}^{k}}{\partial Q_{j}^{k}}-\lambda_{i}^{k}, \\
& \text { if } \quad s_{i j}^{k *}=0 .
\end{aligned}
$$

Assume that the affection utility function associated with a particular location and a single class can depend upon the population associated with every class and each location, that is, compose a vector-function $u=u(Q)$. Also suppose that the cost 
associated with migration between two locations as perceived by a particular class can depend, in general, upon the flow of each class between every pair of locations, i.e., compose an aggregate vector-function $c=c(s)$. Finally, let us form an auxiliary vector of the appropriate size as follows:

$$
d(Q, s):=\left(d_{i j}^{k}(Q, s)\right)
$$

where

$$
d_{i j}^{k}(Q, s):=s_{i j}^{k} v_{i j}^{k-} \frac{\partial u_{i}^{k}}{\partial Q_{i}^{k}}-\sigma_{i j}^{k-} Q_{i}^{k} \frac{\partial u_{i}^{k}}{\partial Q_{i}^{k}}+s_{i j}^{k} v_{i j}^{k+} \frac{\partial u_{j}^{k}}{\partial Q_{j}^{k}}+\sigma_{i j}^{k+} Q_{j}^{k} \frac{\partial u_{j}^{k}}{\partial Q_{j}^{k}} .
$$

Now, we are in a position to formulate the following result established in the previous papers $[5,6]$ :

Theorem 1 A population and migration flow pattern satisfy the equilibrium conditions (8) and (9) if, and only if it solve the variational inequality problem

$$
\left\langle-u\left(Q^{*}\right), Q-Q^{*}\right\rangle+\left\langle c\left(s^{*}\right)-d\left(Q^{*}, s^{*}\right), s-s^{*}\right\rangle \geq 0, \quad \forall(Q, s) \in M .
$$

The existence of at least one solution to the variational inequality (17) follows from the general theory of variational inequalities, under the sole assumption of continuous differentiability of the utility functions $u$ and continuity of migration cost functions $c$, since the feasible convex set $M$ is compact ( $c f$., for example, Kinderlehrer and Stampacchia [9]).

From now on, we omit the superscript $k$ for simplicity purpose. The uniqueness of the equilibrium population and migration flow pattern $\left(Q^{*}, s^{*}\right)$ follows under the assumption that the compound operator

$$
\left(\begin{array}{c}
-u(Q) \\
c(s)-d(Q, s)
\end{array}\right): R^{K \times n} \times R^{K \times n \times(n-1)} \rightarrow R^{K \times n} \times R^{K \times n \times(n-1)},
$$

involving the utility and migration cost functions, is strictly monotone over the feasible set $M$ :

$$
\begin{aligned}
\left\langle\left(\begin{array}{c}
u\left(Q^{1}\right) \\
c\left(s^{1}\right)-d\left(Q^{1}, s^{1}\right)
\end{array}\right)\right. & \left.-\left(\begin{array}{c}
-u\left(Q^{2}\right) \\
c\left(s^{2}\right)-d\left(Q^{2}, s^{2}\right)
\end{array}\right),\left(\begin{array}{c}
Q^{1}-Q^{2} \\
s^{1}-s^{2}
\end{array}\right)\right\rangle>0, \\
& \forall\left(\begin{array}{c}
Q^{1} \\
s^{1}
\end{array}\right) \neq\left(\begin{array}{c}
Q^{2} \\
s^{2}
\end{array}\right),
\end{aligned}
$$

that is,

$$
\begin{aligned}
& -\left\langle u\left(Q^{1}\right)-u\left(Q^{2}\right), Q^{1}-Q^{2}\right\rangle+\left\langle c\left(s^{1}\right)-c\left(s^{2}\right), s^{1}-s^{2}\right\rangle \\
& -\left\langle d\left(Q^{1}, s^{1}\right)-d\left(Q^{2}, s^{2}\right), s^{1}-s^{2}\right\rangle>0, \quad \forall\left(\begin{array}{c}
Q^{1} \\
s^{1}
\end{array}\right) \neq\left(\begin{array}{c}
Q^{2} \\
s^{2}
\end{array}\right) .
\end{aligned}
$$


The latter is a consequence of the following classical result of the Theory of Variational Inequality Problems (see, for example, Kinderlehrer and Stampacchia [8]):

Theorem 2 Consider the variational inequality: Find a $y^{*} \in M \subset R^{m}$ such that,

$$
\left\langle F\left(y^{*}\right), y-y^{*}\right\rangle \geq 0, \quad \forall y \in M
$$

If the operator $F: R^{m} \rightarrow R^{m}$ is strictly monotone over $M$, that is,

$$
\left\langle F\left(y^{1}\right)-F\left(y^{2}\right), y^{1}-y^{2}\right\rangle>0, \quad \forall y^{1}, y^{2} \in M, y^{1} \neq y^{2},
$$

then the variational inequality (21) has at most one solution.

Having established the existence and uniqueness of the (lower level) equilibrium among the potential migrants, we may pass to the concept of the upper level equilibrium among the municipal authorities.

\subsection{Definition of Equilibrium at the Upper Level}

We assume that the affection utility function associated with location $i$ and class $k$ of potential migrants has the form (which is an extension of the linear utility function used in the previous work [8])

$$
u_{i}^{k}\left(Q_{i}^{k}\right)=A_{i}^{k}-\frac{B_{i}^{k}}{R_{i}} Q_{i}^{k}-\beta_{i}^{k}\left(Q_{i}^{k}\right)^{2},
$$

where $A_{i}^{k}>0, B_{i}^{k}>0, \beta_{i}^{k}>0$ are parameters related to the environment facilities for the potential immigrants of group $k$ in location $i$. For instance, the economical sense of $A_{i}^{k}$ could be the average cost of a household in location $i$ in a district where the typical representatives of class $k$ prefer to settle down, while $B_{i}^{k}$ might be interpreted as an inverse affection coefficient for the immigrants of group $k$ : that is, the lower the value of $B_{i}^{k}$, the higher the degree of affection revealed by the average family of the specimen of class $k$ to the growing population of location $i$. Finally, the parameter $R_{i}$ reflects the amount of investment by the authorities of location $i$ into the improvement in the environment for the newcomers and the regular inhabitants: The higher the invested amount, the lower the negative effect of the growing population on the location's attractiveness and affection grade for both the current and potential inhabitants.

Now supposing that the investment volumes $R_{i}>0$ are used as strategies of the players (municipal authorities of the locations involved), it is standard to define an equilibrium state in the (upper level) game.

Definition 3 An investment vector $R^{*}=\left(R_{1}^{*}, \ldots, R_{n}^{*}\right)$ is called the equilibrium at the upper level if for any location $i, i=1, \ldots, n c$, the municipal authority's 
utility function $U_{i}=U_{i}\left(R_{i}, R_{-i}^{*}\right)$ attains its maximum value exactly at $R_{i}=R_{i}^{*}$, if it is assumed that all the rest of the players are stuck to their investment values $R_{-i}^{*}=\left(R_{1}^{*}, \ldots, R_{i-1}^{*}, R_{i+1}^{*}, \ldots, R_{n}^{*}\right)$. Here, the municipality affection utility function $U_{i}=U_{i}(R)$ is the weighted sum of the location's utility functions of all the classes of potential migrants determined below:

$$
U_{i}(R):=\frac{Q_{i}^{1 *}}{Q_{i}^{*}} u_{i}^{1}\left(Q^{*}\right)+\cdots+\frac{Q_{i}^{K *}}{Q_{i}^{*}} u_{i}^{K}\left(Q^{*}\right),
$$

where $Q^{*}$ is the equilibrium of the lower level population values, which (due to Theorems 3.1 and 3.1) exists uniquely for any (fixed) vector of investments $R$ involved into the structure of locations' affection utility functions (23).

\section{Existence of a Bi-level Equilibrium with Consistent Conjectures}

The consistency of conjectures (or, the influence coefficients) arises naturally as an important issue. Indeed, the existence of at least one equilibrium for arbitrary influence coefficients obliges one to select some justified conjectures so that the above concept of the equilibrium make sense. In this section, we propose a concept of consistency and formulate the existence result for the consistent conjectural variations equilibrium (CCEV).

Based upon the consistency criterion proposed in [10], we formulate the following definition. Here, for simplicity, we recall our assumption that the affection utility function for each location $i$ and every potential migrant group $k$ are quadratic of the form $u_{i}^{k}\left(Q_{i}^{k}\right)=A_{i}^{k}-\frac{B_{i}^{k}}{R_{i}} Q_{i}-\beta_{i}^{k}\left(Q_{i}^{k}\right)^{2}$, with $A_{i}^{k}>0, B_{i}^{k}>0, R_{i}>0, \beta_{i}^{k}>0$; next, $a_{i j}^{k}>0$ for each quadratic migration cost function; and finally, conjectures (influence coefficients) are constant with zero elasticity, i.e., $\sigma_{i j}^{k, \pm}=0$, and $v_{i j}^{k+}=v_{i j}^{k-}=v_{i j}^{k}>0$, for all $i, j, k$.

Definition 4 At a lower level equilibrium (LLE) pattern $\left(Q^{*}, s^{*}\right) \in M$, the influence coefficients $v_{i j}^{k} \equiv w_{i j}^{k} \frac{B_{j}^{k}}{R_{j}}, k=1, \ldots, K ; i, j=1, \ldots, n ; i \neq j$, are referred to as consistent, if the following equalities hold:

$$
w_{i j}^{k}=\frac{1}{2 \frac{B_{j}^{k}}{R_{j} \beta_{j}^{k}}+\sum_{\substack{\ell \neq j \\ \ell \neq k}} \frac{1}{w_{\ell j}^{k}+\frac{a_{\ell j}^{k} R_{j}}{B_{j}^{k}}}} .
$$

The LLE with consistent conjectures is called a consistent conjectural variations equilibrium state (CCVES) in application to the above-described human migration model. 
Now, we are in a position to formulate the following existence result.

Theorem 3 Under assumptions A1, A2, and A3, and if all the investment sums are bounded (i.e., $0<R_{i} \leq R, i=1, \ldots, n$ ), then there exists a consistent conjectural equilibrium state (CCVES) in application to the above-described bi-level human migration model.

When proving Theorem 4, we established that certain infinite-dimensional mappings involved in Eq. (4) are continuous and contracting over corresponding compact subsets. This allows one to find, for each fixed group $k$ of potential migrant and each destination location $j$, good approximations for the consistent conjectures (influence coefficients) $v_{i j}^{k} \equiv w_{i j}^{k} \frac{B_{j}^{k}}{R_{j}}, k=1, \ldots, K ; i, j=1, \ldots, n ; i \neq j$, by applying a simple iteration procedure:

$$
w_{i j}^{k,(m+1)}=\frac{1}{2 \frac{B_{j}^{k}}{R_{j} \beta_{j}^{k}}+\sum_{\substack{\ell \neq j \\ \ell \neq i}} \frac{1}{w_{\ell j}^{k,(m)}+\frac{a_{\ell j}^{k} R_{j}}{B_{j}^{k}}}}, \quad m=0,1, \ldots,
$$

with $w_{i j}^{k,(0)}=0, k=1, \ldots, K ; i, j=1, \ldots, n ; i \neq j$.

The convergence of process (26) is established in the following theorem.

Theorem 4 For each fixed group $k$ of potential migrants and every pair locationdestination ( $i, j), i \neq j$, the approximate conjectures (influence coefficients) obtained by formulas (26) converge (as $m \rightarrow \infty$ ) to the unique solution of system (25).

In our future research, we are going to extend the obtained results to the case of not necessarily quadratic affection utility functions and discontinuous conjectures (influence coefficients). However, some of the necessary technique can be developed now, in the case of quadratic utilities and continuous conjectures. To do that, for fixed values of $k$ and $j$, we denote the value of the inverse of the derivative of the affection utility function by

$$
\tau:=\left[\frac{d u_{j}^{k}}{d Q_{j}^{k}}\left(Q_{j}^{k}\right)\right]^{-1}<0,
$$

and rewrite the consistency equalities (25) in a more general form:

$$
w_{i j}^{k}=\frac{1}{-\frac{2}{\tau \beta_{j}^{k}}+\sum_{\substack{\ell \neq j \\ \ell \neq i}} \frac{1}{w_{\ell j}^{k}-\tau a_{\ell j}^{k}}},
$$

where $\tau \in(-\infty, 0]$. When $\tau \rightarrow-\infty$, then the solution of system (28) tends to the unique limit solution $v_{i j}^{k} \equiv w_{i j}^{k} \frac{B_{j}^{k}}{R_{j}}=1, k=1, \ldots, K ; i, j=1, \ldots, n ; i \neq j$. In other words, for all the finite values of the parameter $\tau \leq 0$, we can prove the following result. 
Theorem 5 For each fixed group $k$ of potential migrants and every pair locationdestination $(i, j), i \neq j$, and for any $\tau \in(-\infty, 0]$, there exists a unique solution of Eq. (28) as a collection of continuous functions $w_{i j}^{k}=w_{i j}^{k}(\tau), i, j=1, \ldots, n ; i \neq j$. Furthermore, $w_{i j}^{k}(0)=0$, and $v_{i j}^{k}(\tau) \equiv w_{i j}^{k}(\tau) \frac{B_{j}^{k}}{R_{j}} \rightarrow 1$ as $\tau \rightarrow-\infty$, for all $i, j=1, \ldots, n ; i \neq j$

\section{Conclusions and Future Research}

We investigated a human migration model based upon Kansei engineering principles and involving conjectures of the migration groups concerning the variations in the affection utility function values, both in the abandoned location and in the destination site. To formulate equilibrium conditions for this model, we used the concept of conjectural variation equilibrium (CVE). We established the existence and uniqueness results for the equilibrium in question, and introduced a concept of consistent conjectures (influence coefficients) together with the corresponding CVEs. The theorem guaranteeing the existence and uniqueness of a solution to each consistency system, and therefore the consistent conjectural variation equilibrium state (CCVES), was also proven.

We also notice that the human migration model with conjectural variations can be further extended and examined in the case when constraint (2) is replaced by a weaker condition, as in

$$
Q_{i}^{k} \geq 0, i=1, \ldots, n ; k=1, \ldots, K
$$

which allows us to consider the repeated or chain migration. In this case, the feasible set $M$ ceases to be compact (remaining, however, convex), which makes the use of the general theory of variational inequality problems insufficient to demonstrate the existence of equilibrium. Then, subtler results obtained in Bulavsky et al. [11] and further developed in Isac et al. [4] can be used instead. Indeed, the existence of equilibrium will be guaranteed for various classes of affective utility functions and migration costs that are free of exceptional families of elements (EFE).

Acknowledgements The first author's research activities were financially supported by the R\&D Department (Cátedra de Investigación) CAT-174 of the Instituto Tecnológico y de Estudios Superiores de Monterrey (ITESM), Campus Monterrey, and by the SEP-CONACYT grant CB2008-01-106664, Mexico. The second and the fourth authors were also supported by the SEPCONACyT grant CB-2009-01-127691 and the PAICYT project CE250-09, Mexico. The fourth author was supported by the SEP-CONACyT grant CB-2011-11-169765.

\section{References}

1. Akkoyunlu S, Vickerman R (2001) Migration and the efficiency of European labour markets. Working Paper, Department of Economics, The University of Kent at Canterbury

2. Bulavsky VA, Kalashnikov VV (1994) One-parametric driving method to study equilibrium. Econ Math Methods (Ekonomika i Matematicheskie Metody, in Russian) 30(2):129-138 
3. Bulavsky VA, Kalashnikov VV (1995) Equilibria in generalized Cournot and Stackelberg models. Econ Math Methods (Ekonomika i Matematicheskie Metody, in Russian) 31(3):164-176

4. Isac G, Bulavsky VA, Kalashnikov VV (2002) Complementarity, equilibrium, efficiency and economics. Kluwer Academic Publishers, Dordrecht

5. Kalashnikov VV, Kalashnykova NI, Luévanos R, Uranga C, Méndez M, Luévanos A (2007) Un modelo de migración humana: Experimentos numéricos basados sobre los datos de las tres ciudades Laguneras. Estudios Demográficos y Urbanos 22(3):731-760

6. Kalashnikov VV, Kalashnykova NI, Luévanos R, Uranga C, Méndez M, Luévanos A (2008) Numerical experimentation with a human migration model. European J Oper Res 189(1):208-229

7. Kalashnikov VV, Kalashnykova NI, Chávez Delgadillo LR (2011) Consistent conjectures in a human migration model: definition, existence and computation. Int J Innovative Comput Inf Control 7(4):1949-1957

8. Kalashnikov VV, Kalashnykova NI, Alcorta García MA, Acosta Sánchez YG, KalashnikovVV Jr (2012) Consistent conjectural variations equilibrium in a bilevel human migrationmodel. Int Bus Eco Res J 11(2):195-204

9. Kinderlehrer D, Stampacchia G (1980) An introduction to variational inequalities and their applications. Academic Press, New York

10. Kalashnikov VV, Bulavsky VA, Kalashnykova NI, Castillo FJ (2011) Mixed oligopoly with consistent conjectures. European J Oper Res 210(3):729-735

11. Bulavsky VA, Isac G, Kalashnikov VA (1998) Application of topological degree theory to complementarity problems. In: Migdalas A et al (eds) Multilevel optimization: algorithms and applications. Kluwer Academic Publishers, Dordrecht, pp 333-358 